\title{
Human Umbilical Cord Stem Cells in Chitosan Attenuate Myocardial Injury in Rat Cardiac Infarction
}

\author{
Robert J Henning $\mathrm{MD}^{1^{*}}$, Abraham Khan $\mathrm{MD}^{1}$ and Xiao Wang $\mathrm{MD}^{2}$ \\ ${ }^{1}$ University of South Florida, Tampa, Florida, USA \\ ${ }^{2}$ First Affiliated Hospital, Sun Yat-sen University, Guangzhou, China
}

*Corresponding author: Robert J Henning MD, University of South Florida, 13201 Bruce B. Downs Blvd, Tampa, Florida, USA

\begin{abstract}
Background: Myocardial infarction (MI) is the leading cause of cardiovascular deaths and disability in the industrialized world. Although stem cells have been injected into hearts to limit $\mathrm{MI}$ damage, $<4 \%$ of stem cells remain in the heart for $>1$ hour due to myocardial contractility which causes the rapid egress of the stem cells through the cardiac veins and lymphatics. We hypothesized that stem cells in chitosan gels would remain longer in the heart and therefore be more beneficial in $\mathrm{MI}$ repair.
\end{abstract}

Methods: 100 rats with Mls were divided into Control, Chitosan Gel, human umbilical cord stem cells (hUCBC), or hUCBC in Gel groups for Ml injections. Echocardiograms were done before and at 2, 4, and 8 weeks after injections then random hearts were examined for infarct size and histopathology at each time.

Results: Control infarcts averaged $25 \pm 1 \%, 26.5 \pm 1 \%$ and $27 \pm 1 \%$ of the ventricular area at 2,4 , and 8 weeks. hUCBC in Gel infarcts were smaller than Control, Gel or hUCBC infarcts and averaged $13 \pm 0.9 \%, 11 \pm 0.9 \%$ and $11+0.9 \%(P<0.001$ vs. controls). hUCBC in Gel periinfarct thicknesses averaged $889 \pm 10 \mu \mathrm{m}$ at 4 weeks and were greater than other groups $(P<0.05)$. Arteriole densities in hUCBC in Gels averaged $6.3 \pm 0.2 /$ high power field and were greater than other Groups $(P<0.05)$. LV fractional shortening (FS) averaged $49 \pm 1 \%$ prior to $\mathrm{MI}$ and decreased in Controls to $24 \pm 1.1 \%, 16.8 \pm 1.2 \%$, and 19.9 $\pm 1.1 \%$. hUCBC in Gel FS were greater than other groups and averaged $38 \pm 1.0 \%, 38 \pm 1.0 \%$, and $39 \pm 1.0(P<$ 0.001 vs. controls). hUCBC in Gel LV diastolic diameters were smaller than Gel or hUCBC Groups, averaged $0.68 \pm$ $0.05,0.65 \pm 0.03$, and $0.64 \pm 0.04$ ( $P<0.05$ vs. controls), and were similar to normals.

Conclusion: hUCBC in Chitosan Gel exceed Chitosan or hUCBC in decreasing MI size and LV remodeling and increasing peri-infarct ventricular thickness and neovascularization.

\section{Keywords}

Stem cells, Umbilical cord stem cells, Chitosan, Hydrogels, Myocardial infarction, Infarct size, Left ventricular fractional shortening, Left ventricular remodeling, Neovascularization

\section{Introduction}

Cardiovascular disease is the leading cause of death and disability in people in the United States and imposes an economic burden of over 200 billion dollars per year [1]. Myocardial infarction (MI) is the leading cause of cardiovascular deaths in the United States. Although the mortality rate after $\mathrm{MI}$ has decreased over the last four decades, the incidence of congestive heart failure (CHF) as a complication of MI has significantly increased. With an acute $\mathrm{Ml}$, as many as 1 billion cardiomyocytes can be destroyed [2]. As a consequence, left ventricular (LV) remodeling occurs with progressive extracellular matrix degradation, infarct expansion, scar thinning, LV dilation and eventually the development of CHF [3]. Currently, 5.7 million people in the United States have $\mathrm{CHF}$ and require 30.7 billion dollars per year for health care services and medications [1]. New treatments are urgently needed for acute $\mathrm{MI}$ to limit myocardial damage, LV deterioration and the development of LV dysfunction and congestive heart failure.

In attempts to limit myocardial damage from acute $\mathrm{MI}$, cardiovascular investigators are administering bone marrow and cardiac stem cells to patients with Mls [4]. However, the results of adult autologous bone marrow mononuclear cell/stem cell administration in the Late

Citation: Henning RJ, Khan A, Wang X (2019) Human Umbilical Cord Stem Cells in Chitosan Attenuate Myocardial Injury in Rat Cardiac Infarction. Int J Stem Cell Res Ther 6:061. doi.org/10.23937/2469570X/1410061

Accepted: June 22, 2019: Published: June 24, 2019

Copyright: (c) 2019 Henning RJ, et al. This is an open-access article distributed under the terms of the Creative Commons Attribution License, which permits unrestricted use, distribution, and reproduction in any medium, provided the original author and source are credited. 
Time Trial, the Time Trial, the Swiss Acute Myocardial Infarction and the ALLSTAR (ALLogeneic Heart STem Cells to Achieve Myocardial Regeneration) Trials have been disappointing with no significant differences reported between stem cell treated patients and patients treated with current optimal medical therapy [5-8]. This may be due, in part, to the fact that less than four percent of stem cells injected into the heart actually remain in the heart for more than one hour $[9,10]$. The majority of the stem cells are rapidly expelled from the heart through the coronary veins, lymphatics, or the injection site due to rhythmic contractions of the heart.

We are investigating human umbilical cord blood mononuclear cells (hUCBC), which contain hematopoietic, endothelial and mesenchymal stem cells for the treatment of acute $\mathrm{MI}$ and cardiomyopathy [11-15]. In order to limit or prevent the rapid expulsion of the hUCBC from the heart and limit infarction size and LV remodeling, we are investigating hUCBC in chitosan hydrogels that are injected into the heart [16]. Chitosan is a linear polysaccharide, consisting of D-glucosamine and $\mathrm{N}$-acetyl-D-glucosamine linked by glycosidic bonds that are obtained by treating crustacean shells with sodium hydroxide [17-20]. Chitosan hydrogels consist of a mixture of chitosan, beta-glycerol phosphate, and hydroxyethyl cellulose which are liquid at room temperature but undergo gelation and form a matrix in body tissues at $37^{\circ} \mathrm{C}$. Chitosan gels are biocompatible, non-toxic, bacteriostatic, and are porous $[19,20]$. Chitosan and chitosan hydrogels have been successfully used in drug delivery systems and for repair of skin, bone, cartilage, nerves, liver, and muscle $[20,21]$. We have previously demonstrated in rats with acute Mls that chitosan hydrogels can increase LV wall thickness and decrease LV wall tension, which is a primary determinant of myocardial oxygen requirements, by the Law of LaPlace as applied to the heart (Wall tension $=$ Distending Pressure $x$ Radius/2 $\times$ Wall Thickness). In this manner, chitosan hydrogels can decrease myocardial oxygen requirements and LV infarct size [16]. In the present experiments, we compared the effects of hUCBC in chitosan with the effects of chitosan alone and hUCBC alone in the treatment of acute Mls in rats.

\section{Materials and Methods}

\section{Research animals}

Male Sprague-Dawley rats, weighing 250-350 g, were housed in a temperature-controlled environment with free access to food and water. On the day of surgery, the rats were anesthetized with $5 \%$ Isoflurane by inhalation, intubated with polyethylene tubing, and mechanically ventilated (Harvard Apparatus) with oxygen and 3 to $5 \%$ Isoflurane. The rats were then placed in the right lateral decubitus position on a heating pad. A thoracotomy was performed in the fourth left intercostal space. The pericardium was opened and the left anterior descending coronary artery (LAD) was permanently ligated with 5-0 prolene suture $3 \mathrm{~mm}$ below its origin from the aorta. Myocardial infarction in each rat was confirmed after LAD ligation by discoloration and akinesis of the anterior myocardial wall and ST-segment elevation on the ECG one hour after coronary artery ligation.

Male rats were utilized in the present experiments rather than female rats in order to avoid hormonal changes that might affect LV remodeling. One hundred male rats were randomly divided into four groups for myocardial infarction injections. In each group, the tip of the injection needle was bent 90 degrees in order that the needle remained parallel with the LV myocardial wall and did not enter the LV cavity. Prior to the myocardial wall injection, the syringe connected to the needle was aspirated to ensure the needle had not entered the LV cavity. The control group consisted of rats in which the LAD coronary artery was permanently ligated and, after 1 hour, 0.2-0.5 ml Dulbecco's Modified Eagle Medium (DMEM), was injected around the perimeter of each MI. The infarct + chitosan group consisted of rats that underwent LAD ligation and after 1 hour were given $0.5 \mathrm{ml}$ chitosan in DMEM around the perimeter of each infarction. The infarct $+\mathrm{hUCBC}$ group consisted of rats that underwent $L A D$ ligation and after 1 hour were given $4 \times 10^{6}$ hUCBC in DMEM around the perimeter of each infarction. The infarct $+\mathrm{hUCBC}$ in chitosan group consisted of rats that underwent LAD ligation and after 1 hour were given $4 \times 10^{6}$ hUCBC in $0.5 \mathrm{ml}$ chitosan around the perimeter of each infarction. The chest wall of each rat was then closed with 3-0 Vicryl then 3-0 Prolene sutures in three separate layers and the Isoflurane anesthesia was discontinued. The rats were then continuously monitored for one to two hours until alert and ambulatory. Carprofen $(5 \mathrm{mg} / \mathrm{kg}, \mathrm{SC})$ was given for analgesia every 12 hours for 48 hours then as needed after surgery. The perioperative mortality in this investigation was $18 \%$. The Institutional Review and Animal Use Committees approved all of our protocols and procedures. All experiments complied with the National Institutes of Health guide for the care and use of laboratory animals (NIH Publications No. 8023).

\section{Chitosan hydrogel}

Two hundred mg of chitosan (Sigma), which was 75$90 \%$ deacetylated, was dissolved in $10 \mathrm{ml}$ of distilled water to obtain a $2 \%$ chitosan solution and sterilized using liquid autoclaving. Then $1.15 \mathrm{~g}$ of ultrapure $\beta$-glycerol phosphate (Sigma) was dissolved in $10 \mathrm{ml}$ of distilled water to obtain $11.5 \% \beta$-glycerol phosphate ( $\beta$-GP) and the solution was filter sterilized. Eight $\mathrm{ml}$ of chitosan and $2 \mathrm{ml}$ of $\beta$-GP was then mixed with $2.5 \mathrm{ml}$ of $2.5 \%$ sterile hydroxyethyl cellulose in DMEM $[16,22]$. The product was liquid at room temperature but formed a gel at body temperature $\left(37^{\circ} \mathrm{C}\right)$ in the rat within approximately ten minutes. The gel was gradually degraded over two to four weeks to non-toxic metabolites that were excreted in the urine $[23,24]$. 


\section{hUCBC}

Human umbilical cord blood mononuclear cells or human cord blood were obtained from human cord cell blood banks (CordUse, Cambrex, Cryo-Cell) in order to insure the validity of the results and stored at $-196^{\circ} \mathrm{C}$ in liquid nitrogen. The cord blood was rejected if the maternal blood was positive for the human immune virus, human T-lymphocytic virus, hepatitis, syphilis or cytomegalovirus. The mononuclear fraction of cord blood was obtained by Ficoll density gradient separation and cryopreserved at $-196^{\circ} \mathrm{C}$ in liquid nitrogen. The cryopreserved $\mathrm{hUCBC}$ were thawed at $37^{\circ} \mathrm{C}$ and transferred into centrifuge tubes containing Isolyte ${ }^{\circledR}$ S, pH 7.4 (B. Braun Medical). The cells were washed three times, centrifuged at $1500 \mathrm{rpm}$ for $7 \mathrm{~min}$, the supernatant discarded and the hUCBC viability determined by Trypan Blue dye exclusion technique. The hUCBC viability were greater than $95 \%$. The hUCBC were not propagated in culture flasks. Each dose of hUCBC administered was adjusted to deliver a total of $4 \times 10^{6}$ Trypan Blue-negative hUCBC by a precisely calibrated syringe. All cells were injected into the peri-infarct area of the myocardium within two hours of preparation.

The hUCBC contained 1 to $2 \% \mathrm{CD}^{+} 4^{+}$cells and approximately $1 \%$ SH2 (CD 105) cells as determined by fluorescent antibodies to $\mathrm{CD} 34$ and $\mathrm{SH} 2$ cell antigens that were obtained from Invitrogen and Osiris, respectively, and fluorescent activated cell sorting cytometry (Becton Dickinson) in our facility. A computer (Apple) was used for initial cell analysis. Cell Quest (Becton Dickinson), was used for cell acquisition. The data was then analyzed with FlowJo (Tree Star) on a computer (Apple). We did not detect any significant differences among the hUC$B C$ supplied by the three vendors. Immune suppression therapy was not given to any rat that received hUCBC.

\section{hUCBC plus chitosan hydrogel}

$4 \times 10^{6}$ Trypan Blue-negative hUCBC were mixed with $0.5 \mathrm{ml}$ of chitosan hydrogel and the mixture was injected around the periphery of each myocardial infarction in the rats randomized to receive hUCBC in chitosan. In pilot studies, we determined that hUCBC remained viable in chitosan hydrogels when the mixture was cultured in DMEM for four or more weeks.

\section{Infarct size}

Hearts were harvested from randomly selected anesthetized rats from each group of rats at baseline then at two, four, and eight weeks after myocardial infarction. The ventricles of each heart were cut into four separate 2.5 millimeter slices parallel to the atrioventricular sulcus. Each biventricular slice was rinsed in $0.9 \% \mathrm{NaCl}$. Thereafter, each biventricular slice was totally immersed and turned every five minutes in a dark room for forty-five minutes in $1 \%$ triphenyltetrazolium chloride (TTC) solution containing $0.2 \mathrm{M}$ Tris at room temperature $[13,25]$. The myocardial slices were then stored in $10 \%$ formalin for 24 hours. The ventricular slices were then rinsed in saline and photographed with a digital camera. Triphenyltetrazolium forms a red precipitate in the presence of intact dehydrogenase enzymes in normal myocardium whereas infarcted and injured myocardium lacks these enzymes and appears white to light pink in color within 30 minutes after acute coronary occlusion. Tetrazolium does not stain autolytic myocardium and has a diagnostic efficiency of $88 \%$ for infarcted myocardium [25]. Computer imaging software (ImagePro Plus) was utilized for planimetry of the area of the infarcted myocardium and the area of normal myocardium in the right and left ventricles. The area of infarction and the area of normal right and left ventricles for each heart slice were summed to determine total infarct area and the total right and left ventricular area for each heart. Infarct size was then expressed as infarct area divided by total left plus right ventricular muscle area. All measurements were done in duplicate and the results were averaged. Measurements were also made with a Vernier micrometer of the region of the ventricle between the area of grossly normal tissue and grossly infarcted tissue in each heart slice and the measurements averaged.

\section{Histopathology}

Pathologists, who were unaware of the treatment for each rat, examined heart tissue from randomly selected rats from each group at baseline, 2, 4, and 8 weeks. Ventricular tissue in paraffin was deparaffinized and hydrated with water. The tissue was then stained with hematoxylin and eosin, Weigert's Iron-Hematoxylin and Safranin-O solution [26], or with antibodies to human nuclear antigen (Millipore) or to von Willebrand antigen (Abcam). The slides were examined and the sections of each ventricle photographed with a digital camera. The arteriole density was determined in LV sections stained with antibodies to von Willebrand antigen. Ten high magnification microscopic fields spanning the border zone of the infarct of each rat heart, defined as the region of the ventricle between the area of grossly normal tissue and grossly infarcted tissue, were randomly chosen. Vessels with visible lumens and endothelium positive for von Willebrand antigen were counted and expressed as the mean number of vessels/high power field (HPF).

\section{Echocardiography}

M-mode and two-dimensional transthoracic echocardiograms were obtained on rats prior to myocardial infarction and then at two, four, and eight weeks after myocardial infarction. This technique is accurate and reproducible in normal rats and rats with myocardial infarction [27]. Echocardiographic systems (Siemens/Acuson) with 7.5 MHz transducers were used. These systems have a resolution of $0.5 \mathrm{~mm}[27,28]$. Rats were lightly anesthetized with 2-3\% Isoflurane and then underwent echocardiographic examinations. Two dimensional and M-mode echocardiograms were obtained at the level of 
the LV papillary muscles and were recorded on the computer hard disc of the echocardiographic system and on compact discs. A minimum of five cardiac cycles were measured and the values averaged for each rat. An investigator and a sonographer, who were unaware of the rats' treatment, analyzed all the echocardiographic images. Left ventricular end-diastolic diameter, which was measured at the onset of the electrocardiographic QRS complex, was measured from the parasternal long-axis or apical views. The left ventricular end-systolic diameter was measured after aortic valve closure or measured from the frame in which the left ventricular chamber dimension was the smallest. Left ventricular fractional shortening was determined from LV End-Diastolic diameter (LVED) minus Left Ventricular End-Systolic diameter (LVES) divided by LVED $\times 100$.

\section{Statistical Analysis}

All results are expressed as the mean \pm SEM Differences among groups were tested by analyses of variance (ANOVA). The difference between the two groups was tested by a Student's t-test. The Bonferroni modification of the t-test was used for repeated statistical analyses for planned comparisons. Tukey's procedure was used for post-hoc comparisons. A value of $p<0.05$ was judged to be significant.

\section{Results}

\section{Infarct size}

Hearts were harvested from randomly selected rats from each of the groups at two, four, and eight weeks after myocardial infarction. The infarct areas as a percentage of the total ventricular area in the DMEM Control treated rats were significantly larger than the infarct areas in the Gel, hUCBC, and hUCBC in Gel group at two, four and eight weeks after permanent LAD coronary artery ligation. The infarct areas in the DMEM Group averaged $25 \pm 1 \%$ at 2 weeks, $26.5 \pm 1 \%$ at 4 weeks and $27 \pm 1 \%$ at 8 weeks. In the Gel Group, the infarct areas averaged $16 \pm 1 \%$ at two weeks, $14 \pm 1 \%$ at 4 weeks, and $14 \pm 1 \%$ at 8 weeks (all $p<0.001$ in comparison with the DMEM Group). The infarct areas in the hUCBC group were similar to the Gel Group and averaged $17 \pm 1 \%$ at two weeks $(P<0.01), 14.5 \pm 0.9 \%$ at 4 weeks and $13.8 \pm$ $0.8 \%$ at 8 weeks (both $\mathrm{P}<0.001$ in comparison with the DMEM Group).

The largest reductions in infarct area were seen in the hUCBC in Gel Group where the infarct areas average $13 \pm 0.9 \%$ at 2 weeks, $11 \pm 0.9 \%$ at 4 weeks and $11 \pm 0.7 \%$ at 8 weeks (all $P<0.001$ in comparison with DMEM). In addition, the relative difference in infarct areas in the hUCBC + Gel group were more than $18 \%$ smaller than the infarct areas in either the Gel or the hUCBC Group ( $P$ $<0.05$ ) at each time. (See Figure 1). Representative myocardial infarctions from rats from the DMEM Group, Gel, hUCBC, and hUCBC in Gel Groups are shown in Figure 2.

\section{LV border wall thickness}

The end-diastolic LV wall thickness in the normal rat averaged $1295 \pm 27$ microns. The LV border zone thickness post-infarction in the DMEM Group averaged $380 \pm 10$ microns. In contrast, the LV wall thickness in the border zones averaged $589 \pm 12$ microns in the Gel Group, $620 \pm 15$ microns in the hUCBC Group and 889 \pm 10 microns in the hUCBC in Gel Group (all $P<0.05$ in comparison with DMEM). The wall thickness in the hUCBC in Gel Group was significantly greater than the Gel and the hUCBC Group ( $P<0.05)$.

The chitosan hydrogel, the hUCBC or the hUCBC in chitosan did not cause inflammatory cell infiltration or

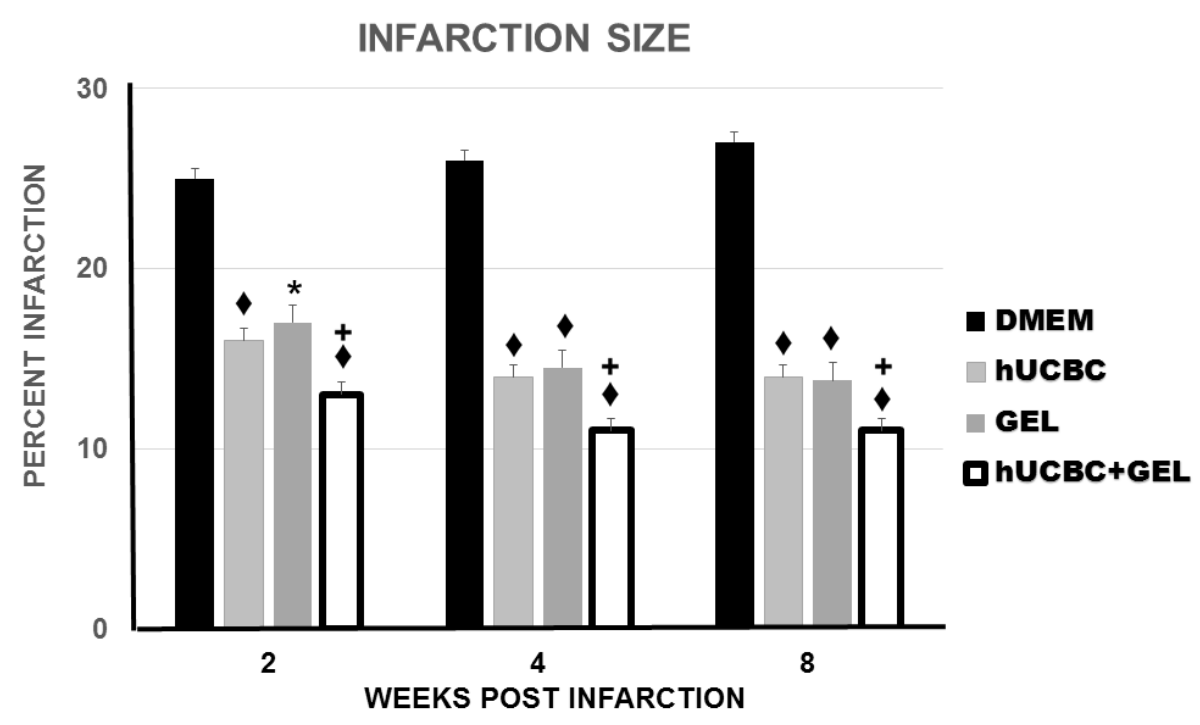

Figure 1: Infarct Size reduction with chitosan hydrogel, hUCBC, and hUCBC in Hydrogel in comparison with rat infarctions treated with DMEM. The smallest infarctions occurred in the hUCBC in chitosan hydrogel Group. Five to six rats from each Group at each time were randomly chosen for infarct size determinations.

$\downarrow \leq 0.001$ and * $\leq 0.01$ in comparison with DMEM. $+<0.05$ in comparison with Gel alone or hUCBC alone. 


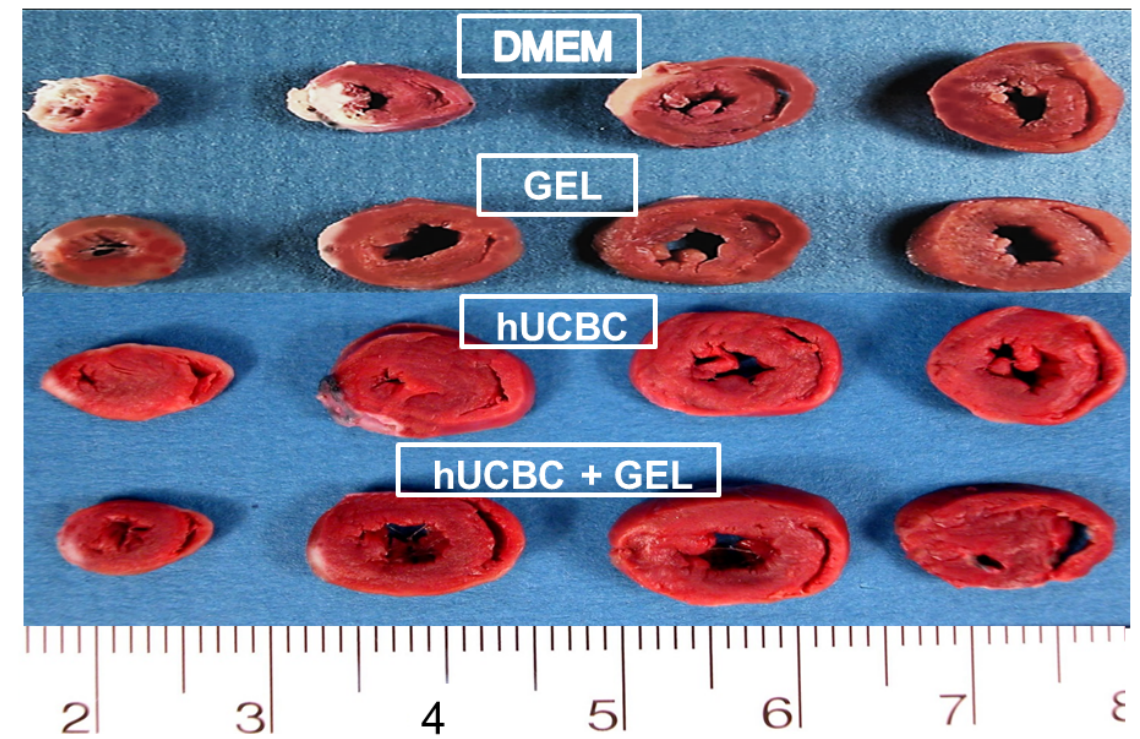

Figure 2: Representative myocardial infarctions from the DMEM Group, the Chitosan Hydrogel Group, the hUCBC Group, and the hUCBC in chitosan hydrogel Group. White areas indicate infarcted myocardium. The smallest infarction occurred in the hUCBC in chitosan hydrogel treated heart.

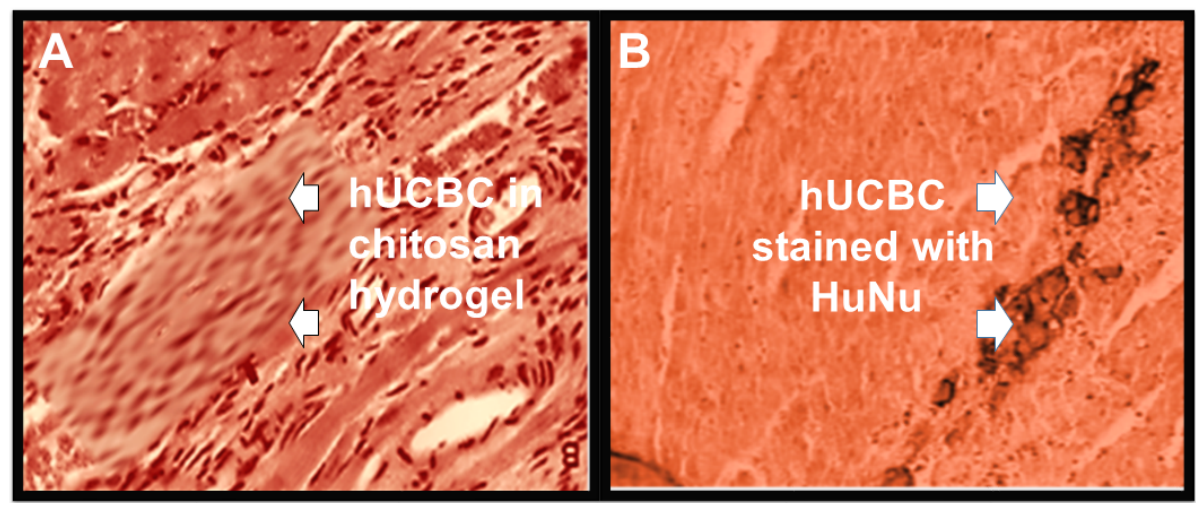

Figure 3: A) Histopathological section of a left ventricular myocardial infarction with hUCBC in chitosan hydrogel at approximately 2 weeks post myocardial infarction; B) hUCBC stained with antibodyt to human nuclear antigen at 3 to 4 weeks post myocardial infarction.

granuloma formation in the heart. There was no gross anatomical or histological evidence of immune rejection in rats that received hUCBC.

\section{Cell retention}

Chitosan persisted in the left ventricular myocardium for two to four weeks (See Figure 3A). At 3-4 weeks, hUCBC were detected in the myocardium of rats treated with hUCBC in Chitosan Group. See Figure 3B. In contrast, no hUCBC were identified in the rat myocardium in the hUCBC Group at 3-4 weeks after the acute infarction. However, hUCBC were detected in the spleens, livers, and lungs of the hUCBC in DMEM Group. We did not observe cardiac myocytes that stained with HuNu or cardiac myocytes with early myofibrillar organization or early $Z$ band development that might indicate hUCBC transdifferentiation to myocytes.

\section{Vascularization}

Vessel density in the border zones of myocardial infarction after 8 weeks of observation and treatment were: $3.0 \pm 0.3 /$ High Powered Field (HPF) in the DMEM treated rats, $4.7 \pm 0.9 / \mathrm{HPF}$ in the Gel only group, $5.3 \pm$ $0.4 / \mathrm{HPF}$ in the hUCBC treated rats, and $6.3 \pm 0.2 / \mathrm{HPF}$ in the hUCBC in Gel group, which was significant at $P<0.05$ in comparison with the DMEM group. See Figure 4.

\section{Left ventricular remodeling}

Left ventricular end-diastolic diameter (LVED) and end-systolic diameter (LVES) in each rat treatment group after MI were used as a measure of LV remodeling. The LV end-diastolic diameters in the rats averaged $0.61 \pm 0.05 \mathrm{~cm}$ prior to MI. After infarction, the LVED significantly increased in the DMEM treated rats to 0.88 $\pm 0.03 \mathrm{~cm}$ at two weeks then $0.89 \pm 0.01 \mathrm{~cm}$ at 4 weeks, and $0.92 \pm 0.05 \mathrm{~cm}$ at 8 weeks (all $P<0.001$ in comparison with normal LVED). See Figure 5. In contrast, the LVED and LV remodeling were significantly less in the chitosan treated rats. In comparison with rats treated with DMEM, the LVED in the chitosan treated rats aver- 


\section{VASCULARIZATION POST INFARCTION}

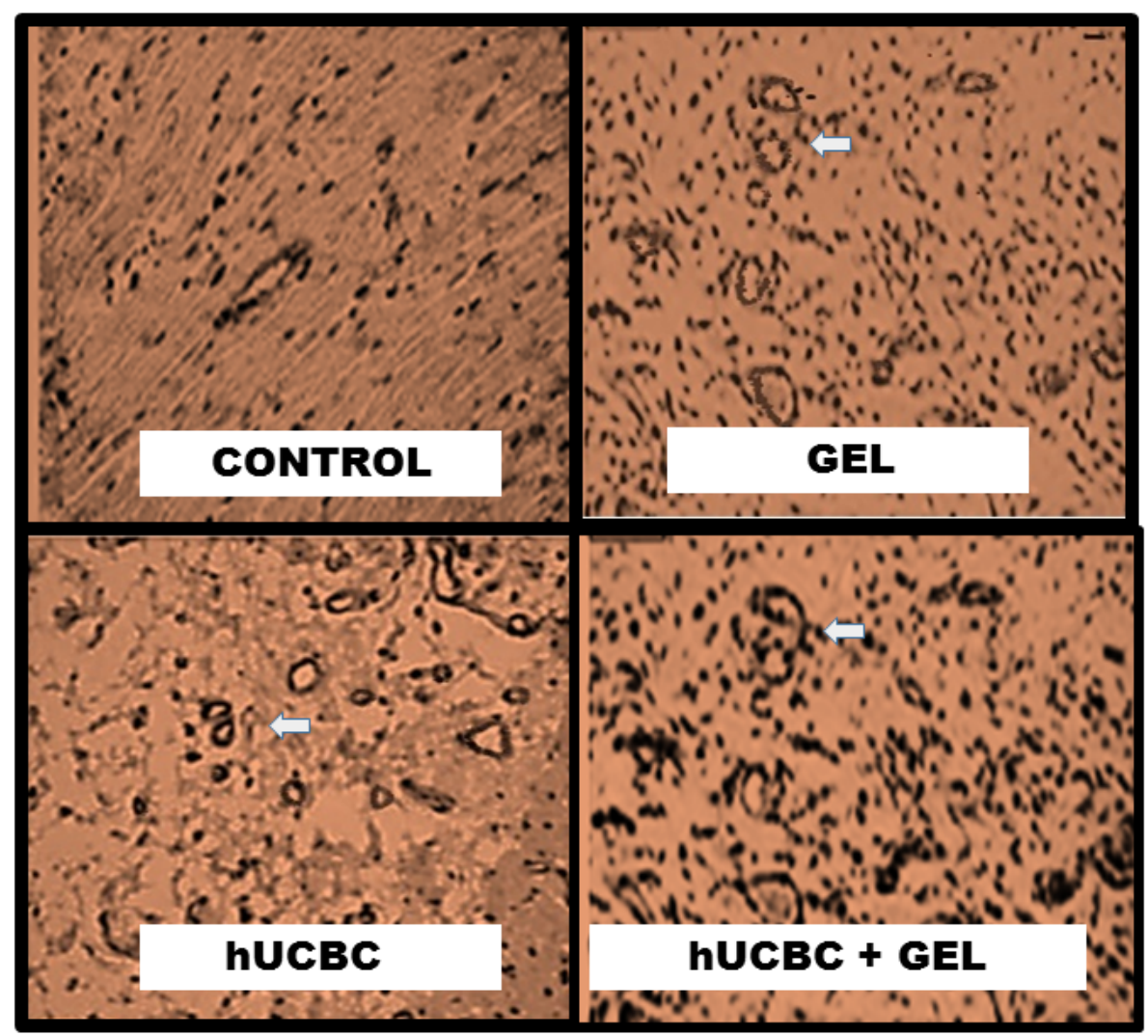

Figure 4: Representative samples of arteriole density taken from rats treated with DMEM, chitosan hydrogels, hUCBC, and hUCBC in chitosan hydrogels at 8 weeks. Rats treated with hUCBC in chitosan hydrogels had significantly more arterioles in the infarct border zone than rats treated with DMEM, chitosan hydrogels, or hUCBC.

\section{END DIASTOLIC DIAMETER}

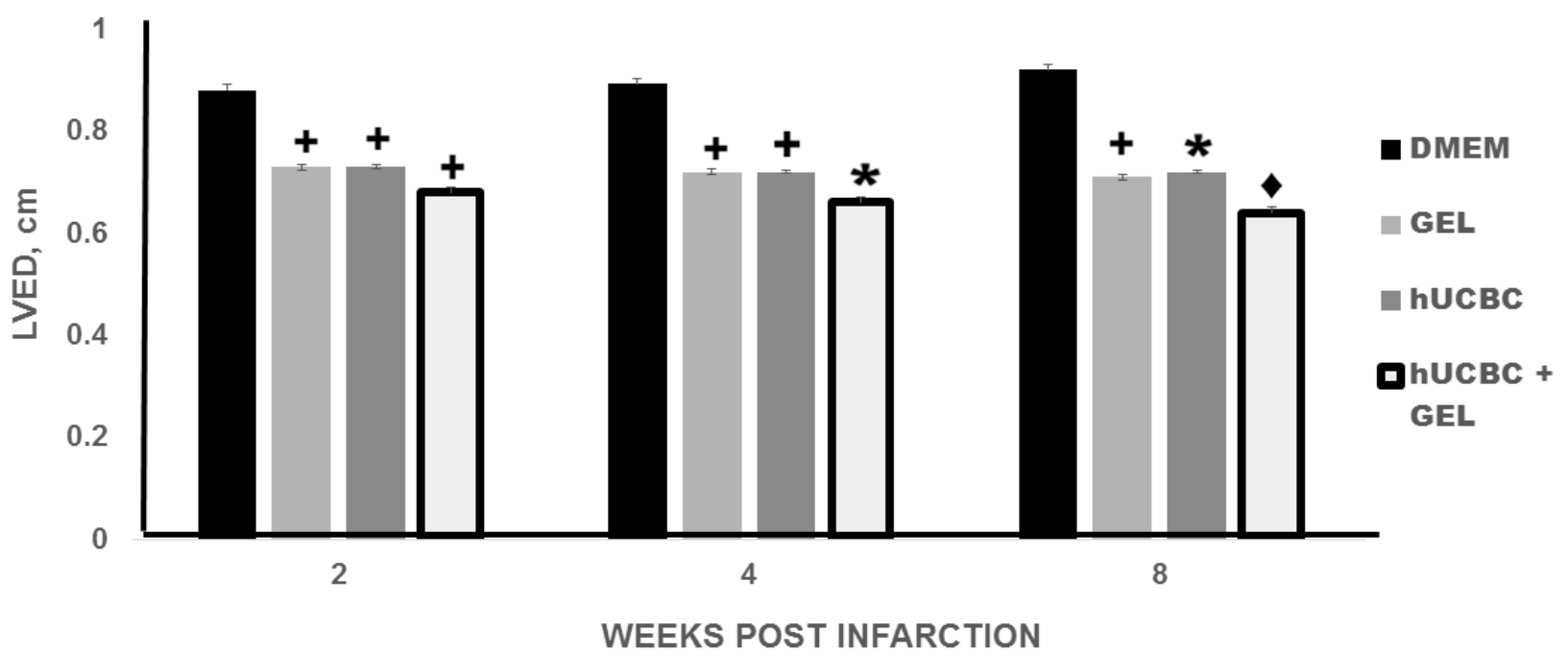

Figure 5: LV End-Diastolic Diameters in rats with chitosan hydrogel, hUCBC, and hUCBC in chitosan hydrogel in comparison with rat infarctions treated with DMEM. A minimum of five to six rats from each Group at each time were randomly chosen for echocardiographic determinations.

$\bullet \leq 0.001,{ }^{*} \leq 0.01$ and $+<0.05$ in comparison with DMEM.

aged $0.74 \pm 0.01 \mathrm{~cm}$ at 2 weeks $(P<0.05), 0.73 \pm 0.03 \mathrm{~cm}$ at 4 weeks $(P<0.05)$, and $0.72 \pm 0.05 \mathrm{~cm}$ at 8 weeks $(P<$ 0.05). See Figure 5.

Injection of hUCBC also limited LV dilation and re- modeling after acute myocardial infarction. In comparison with the rats treated with DMEM, the LVED in the hUCBC Group averaged $0.74 \pm 0.04 \mathrm{~cm}$ at 2 weeks ( $P<$ $0.05), 0.72 \pm 0.05 \mathrm{~cm}$ at 4 weeks $(p<0.05)$, and $0.73 \pm$ 
$0.03 \mathrm{~cm}$ at 8 weeks $(P<0.01)$. The largest reductions in LVED occurred in the rats treated with hUCBC in Gel. In this Group, the LVED averaged $0.68 \pm 0.05$ at 2 weeks $(P<0.05), 0.65 \pm 0.03$ at 4 weeks $(P<0.01)$, and 0.64 \pm 0.04 at 8 weeks $(P<0.001$ in comparison with the DMEM group). In addition, the LVED were smaller than the corresponding diameters in the Gel Group and the hUCBC Group and were not significantly different from the normal control LVED.

The LV end-systolic diameters (LVES) in the rats prior to infarction averaged $0.35 \pm 0.05 \mathrm{~cm}$. Permanent occlusion of the left anterior coronary artery significantly increased the LVES at 2 weeks in the DMEM treated rats to $0.67 \pm 0.03 \mathrm{~cm}(P<0.05)$. See Figure 6 . Thereafter the end-systolic diameters in the DMEM Group averaged $0.68 \pm 0.02 \mathrm{~cm}$ at 4 weeks, and $0.69 \pm 0.05 \mathrm{~cm}$ at 8 weeks (all $P<0.001$ in comparison with the normal rat LVES). In contrast, injection of chitosan hydrogel limited increases in the LV end-systolic diameters and limited LV remodeling. In comparison with the DMEM treated rats at each time, the LVES in the chitosan treated rats averaged $0.47 \pm 0.05 \mathrm{~cm}$ at 2 weeks $(P<0.01), 0.48 \pm 0.05$ $\mathrm{cm}$ at 4 weeks $(P<0.01), 0.44 \pm 0.01 \mathrm{~cm}$ at 8 weeks $(P$ $<0.001)$. Myocardial injection of hUCBC also limited the deterioration of LV systolic function in comparison with the DMEM Group at each time. The LVES in the hUCBC averaged $0.50 \pm 0.04 \mathrm{~cm}$ at 2 weeks $(P<0.05), 0.50 \pm$ $0.03 \mathrm{~cm}$ at 4 weeks $(P<0.05)$, and $0.48 \pm 0.04 \mathrm{~cm}$ at 8 weeks ( $P<0.01$ in comparison with DMEM Group). However, the smallest increases in LVES occurred in the hUCBC in Gel Group where the LVES averaged $0.40 \pm$ $0.04 \mathrm{~cm}$ at 2 weeks $(P<0.01), 0.35 \pm 0.03 \mathrm{~cm}$ at 4 weeks $(P<0.001)$, and $0.34 \pm 0.04 \mathrm{~cm}$ at 8 weeks $(P<0.001)$ in comparison with the DMEM Group. In addition, the LVES were smaller than the corresponding diameters in the Gel Group and the hUCBC Group ( $P<0.05)$ and were not significantly different from the normal control LVES. See Figure 6.

\section{Left ventricular contractility}

Left ventricular fractional shortening (FS) measurements, determined by echocardiography, were used as a measure of LV contractility prior to rat myocardial infarction and then at two, four, and eight weeks after infarction. See Figure 7. Left ventricular FS averaged 49 $\pm 1 \%$ in the rats prior to infarction. In the DMEM group, the left ventricular FS significantly decreased after acute infarction and averaged $24 \pm 1.1 \%$ at 2 weeks. $16.8 \pm$ $1.2 \%$ at 4 weeks, and $19.9 \pm 1.1 \%$ at 8 weeks (all $\mathrm{P}<$ 0.001 in comparison with normal rat FS). The FS measurements in the Gel, hUCBC, and hUCBC in Gel rats were significantly greater than the measurements in the rats in the DMEM group. In the Gel Group, the FS measurements averaged $33.1 \pm 0.9 \%$ at 2 weeks post-infarction, $33.5 \pm 1.1 \%$ at 4 weeks, and $34 \pm 0.9 \%$ at 8 weeks (all $\mathrm{P}<0.001$ in comparison with the DMEM group), See Figure 7. The FS measurements in the hUCBC Group were similar to the hydrogel group and averaged $31 \pm$ $1.1 \%$ at 2 weeks, $32 \pm 1.2 \%$ at 4 weeks and $32 \pm 0.9 \%$ at 8 weeks (all $p<0.001$ in comparison with DMEM). The hUCBC in Gel Group showed the smallest decrease in FS in comparison with the normal rats and averaged $38 \pm$ $1.0 \%$ at 2 and 4 weeks and $39 \pm 1.0$ at 8 weeks (all $P<$ 0.001). In addition, the FS measurements in the hUCBC in Gel group were greater than the measurements in the Gel Group (all P < 0.01) and the hUCBC Group (all P < 0.001 ) at each time. See Figure 7.

\section{Discussion}

The present investigation demonstrates that hUCBC in chitosan hydrogels can significantly limit acute MI size

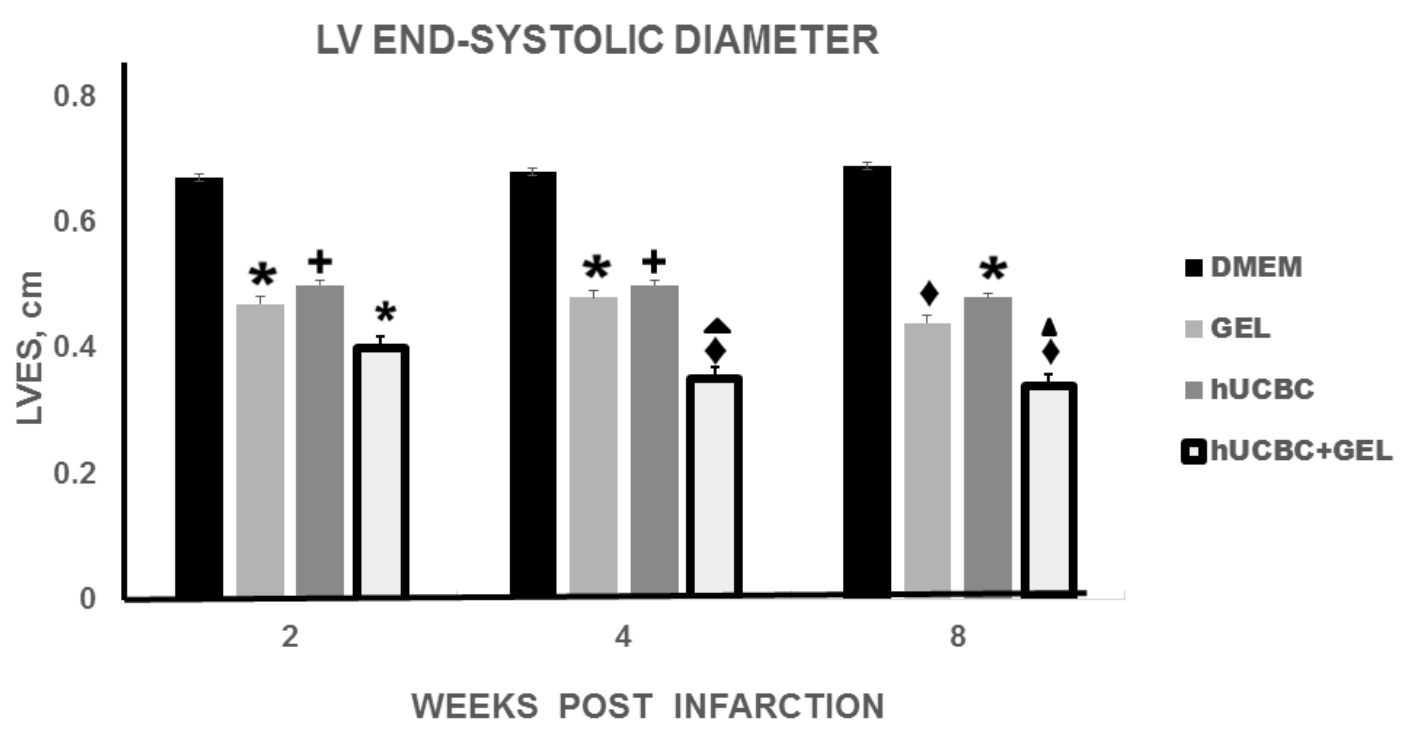

Figure 6: Left Ventricular End Systolic Diameters in rats with chitosan hydrogel, hUCBC, and hUCBC in chitosan hydrogel in comparison with rat infarctions treated with DMEM. A minimum of five to six rats from each Group at each time were randomly chosen for echocardiographic determinations.

$\bullet \leq 0.001,{ }^{*} \leq 0.01$ and $+<0.05$ in comparison with DMEM. $\Delta 0.05$ in comparison with Gel and hUCBC. 


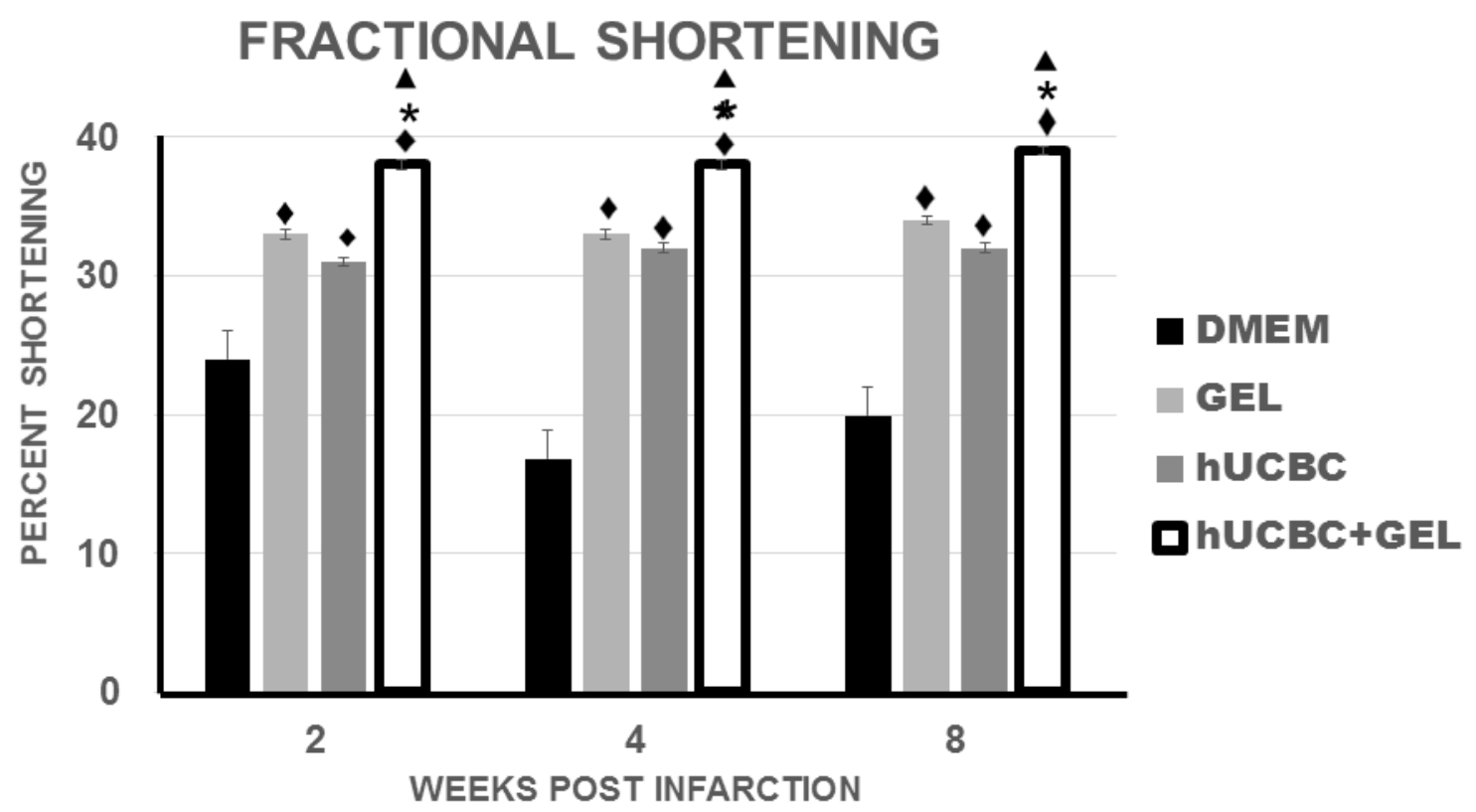

Figure 7: Left ventricular fractional shortening with chitosan hydrogel, hUCBC, and hUCBC in chitosan hydrogel in comparison with rat infarctions treated with DMEM. The fractional shortening measurements in the hUCBC in Gel group were greater than the measurements in the Gel Group and the hUCBC Group at each time. A minimum of five to six rats from each Group at each time were randomly chosen for echocardiographic determinations.

$\downarrow \leq .001$ in comparison with DMEM; * $\leq 0.01$ in comparison with Gel alone and $\mathbf{\Delta}<0.001$ in comparison with hUCBC alone.

and LV remodeling and increase the thickness and the vascularity of the LV infarct border zone. The beneficial effects of hUCBC in chitosan exceed the effects observed with either chitosan or hUCBC alone.

\section{Chitosan hydrogels}

Hydrogels made from chitosan, which are liquid at room temperature, are amendable to cardiac delivery by direct injection into the myocardium during cardiac surgery or by injection into the LV endocardium during cardiac catheterization. The gel is biocompatible and porous to the diffusion of nutrients and oxygen in the myocardium and can create a protective scaffold for stem cells in the myocardium without causing significant LV remodeling. The deacetylation of chitosan can be adjusted to optimize the degradation rate of the gel in the myocardium [16-18]. Chitosan that is $75-90 \%$ deacetylated, which was used in the present experiments, is biodegradable over two to four weeks but chitosan that is $>95 \%$ deacetylated is significantly more resistant to degradation in the myocardium $[19,20]$. The gels are broken down in the myocardium by lysozymes and $\mathrm{N}$-acetyl-beta-D-glucosaminidases into biocompatible oligosaccharides, which are non-toxic metabolites that are excreted in the urine $[17,29,30]$.

Chitosan and chitosan hydrogels are approved by the US Food and Drug Administration for use in tissue engineering and drug delivery and are currently used in skin, liver, blood vessel, nerve, bone, and cartilage repair $[19,31]$.

We propose that the beneficial effects of chitosan in the reduction of infarct size and the decrease in LV remodeling is due to four possible mechanisms: 1) An increase in LV myocardial wall structural support and stiffness that decreases myocardial wall stress and LV remodeling; 2) A decrease in the deleterious effects of reactive oxygen species; 3) An acceleration of infarct scar formation; and 4) A stimulation of myocardial angiogenesis.

In the present investigation, chitosan hydrogel in the myocardial wall limited any significant increase in LV end-diastolic volume, end-systolic volume, and LV remodeling after $\mathrm{MI}$ in contrast to infarcted rat hearts treated with only DMEM. This finding suggests that the chitosan induced increase in LV wall thickness/stiffness reduced LV wall tension or stress and, in this manner, decreased myocardial oxygen requirements, prevented ischemic and injured myocytes from becoming necrotic myocytes, and limited LV chamber dilation and LV remodeling. In this regard, chitosan's increase in LV structural support is directly dependent on the location of the chitosan injection in the LV myocardium, the volume of the hydrogel added to the myocardium and the relative stiffness of chitosan in the myocardium. A gel volume of 0.5-1.5 ml into the perimeter of a MI can increase LV myocardial stiffness by $20 \%$ [32-34]. This gel volume can reduce the infarct border zone myocardial fiber stresses by 18 to $20 \%$ from mean LV end-systolic levels of 212 $\mathrm{mmHg}$ to near normal levels of $175 \mathrm{mmHg}$, which are similar to fiber stresses in remote non-infarcted myocardium [33]. The increase in wall thickness and the resultant reduction in LV wall stress is associated with a decrease in myocardial wall oxygen requirements, LV myocyte necrosis, infarct size, and LV remodeling $[33,34]$. 
Chitosan hydrogels injected into the perimeter of a $\mathrm{Ml}$ can also stimulate the migration of fibroblasts and the resultant fibroblast deposition of type III collagen in the perimeter of the $\mathrm{Ml}$ and, in this manner, can limit the extravasation of death-promoting free radicals from the infarction into the surrounding non-infarcted myocardium. Chitosan can also accelerate the formation and maturation of an infarct scar by stimulating fibroblast migration, collagen deposition and vascularization in the infarct [24,35-43]. In addition, the cross-linkage of chitosan hydrogel glycosidic bonds can contribute to infarct scar contracture [37] Chitosan-induced fibroblast migration into the infarction and resultant collagen deposition also plays an important role in maintaining ventricular shape and size and limiting remodeling due to the relatively rigid structure of collagen that is in close contact with all the other cellular and non-cellular components of the myocardium [44].

Several mechanisms may account for the angiogenesis that we observed in the chitosan treated Mls. Chitosan can release glucosamine and $\mathrm{N}$-acetylglucosamine oligomers and monomers that can recruit native vascular endothelial progenitor cells and can bind these progenitor cells in the infarct perimeter for angiogenesis [35]. In addition, chitosan hydrogel can modulate the proliferation of vascular endothelial cells in the infarct for angiogenesis [36].

Chitosan can also bind platelets and enhance the release of angiogenic growth factors from platelets, which can chemoattract vascular endothelial cells into a MI for angiogenesis [39]. In addition, chitosan's cationic nature causes electrostatic interactions with anionic proteoglycans that attract and retain angiogenic growth factors in an infarction [40]. Chitosan can also form complexes with hyaluronates and heparin sulfates, which in turn bind stromal cell-derived factor-1, fibroblast growth factors, and platelet-derived growth factors that can mediate vasculogenesis and angiogenesis $[38,41]$. Chitosan gel can also protect vascular cells from necrosis due to free oxygen radicals during acute $\mathrm{MI}[16,42]$.

In summary, we propose that chitosan's increase in LV myocardial wall structural support and stiffness, acceleration of infarct scar formation, and stimulation of myocardial angiogenesis best explain the decrease in infarct size and LV remodeling that we observed in the rat infarctions treated with chitosan hydrogels.

\section{Human umbilical cord blood stem cells}

One milliliter of human umbilical cord blood that is obtained at the time of full-term infant delivery contains between 1000 and 10,000 multipotent stem/progenitor cells and approximately 8000 primitive hematopoietic progenitor cells $[11,45]$. Moreover, umbilical cord blood can be frozen for 20 or more years yet permit the recovery of $94 \%$ of the viable primitive cells for transplantation [46].
In the present experiments, hUCBC did not stimulate an inflammatory response in the myocardium. In this regard, hUCBC do not express class II HLA antigens $[45,47]$. In addition, little or no cytotoxic activity is generated with cord blood after allogeneic stimulation because human umbilical cord blood lymphocytes are immature and produce significantly smaller amounts of cytokines and immunoglobulins than adult lymphocytes $[14,45]$. The immaturity and diminished cytotoxicity of cord blood lymphocytes explain the absence in the present experiments of immunological reactions in the hUCBC treated rats during the 8 weeks of observation.

Cord blood endothelial and mesenchymal stem cell peroxidases and catalases limit damage from free radicals and allow the stem cells to survive and maintain their potency in the ischemic and infarcted myocardium $[48,49]$. The hUCBC-induced limitation of myocardial damage and LV remodeling in infarcted myocardium in the present experiments is best explained by the release of growth factors, anti-inflammatory cytokines, nitric oxide, and inhibitors of metalloproteinases. In this regard, we have previously shown that hUCBC secrete angiopoietin, hepatocyte growth factor, anti-inflammatory IL-10, insulin growth factor, placental growth factor, vascular endothelial growth factor, angiogenin, stem cell factor, and tissue inhibitors of metalloproteinases $[50,51]$. Moreover, this paracrine action of hUC$\mathrm{BC}$ is significantly increased during severe hypoxia (i.e. $1-4 \%$ oxygen) with 50 to $100 \%$ or more increases in the secretion of anti-inflammatory cytokines and growth factors which can suppress inflammatory cytokines and inflammatory cell infiltration in ischemic and infarcted myocardium $[50,51]$. In this regard, we have shown that hUCBC paracrine factors can suppress by as much as $50 \%$ the expression of the inflammatory cytokines tumor necrosis factor-alpha, monocyte chemoattraction protein, and macrophage inflammatory protein in acute MIs [14]. hUCBC can also limit caspase expression and the caspase cascade which contributes to myocardial inflammation and programmed cell death after coronary artery occlusion [14]. In addition, hUCBC can limit in acute Mls the myocardial infiltration and proliferation of neutrophils by 65 to $75 \%$ and lymphocytes by more than $50 \%[14,52]$.

An additional mechanism whereby hUCBC can be beneficial in ischemic myocardium is by stimulating myocardial angiogenesis. Vascular endothelial progenitor cells are normal components of umbilical cord blood that can promote angiogenesis by releasing proangiogenic growth factors such as vascular endothelial growth factor, angiogenin, and angiopoietin [50,51]. Endothelial progenitor cells can also participate in vasculogenesis, the de novo formation of blood vessels, or integrate into the walls of blood vessels in the periphery of injured tissues and thereby increase capillary density in ischemic myocardium [53]. 
In summary, the effects of hUCBC in significantly decreasing myocardial infarct size and LV remodeling is best explained by paracrine actions and by stimulating angiogenesis in the periphery of the myocardial infarctions.

\section{hUCBC and chitosan hydrogels}

The beneficial effects of hUCBC in chitosan can be best explained by the prolonged retention of $\mathrm{hUCBC}$ in the infarcted myocardium by the chitosan gel. hUCBC secrete angiopoietin, hepatocyte growth factor, anti-inflammatory IL-10, insulin growth factor, placental growth factor, vascular endothelial growth factor, angiogenin, stem cell factor, and tissue inhibitors of metalloproteinases which can be beneficial in limiting inflammation and myocyte injury and stimulating neovascularization [50,51]. Retention of hUCBC in chitosan gels permitted prolonged hUCBC paracrine actions in the infarctions which decreased myocardial inflammation, prevented ischemic and injured myocytes from becoming necrotic myocytes, increased myocardial neovascularization, and facilitated infarct healing more than either hUCBC or chitosan alone.

Chitosan hydrogels as a vehicle for hUCBC were utilized because of the gel's beneficial effects in the treatment of Mls [22], the gel's ability to provide a protective scaffold for stem cells $[44,54,55]$, accelerate infarct healing [56-59] and the ability to optimize the degradation time of the hydrogel in order to prolong hUCBC paracrine effects in MIs [18]. The deacetylation of chitosan can be adjusted to optimize the degradation rate of the gel in the myocardium [16-18].

The significant increases in LV myocyte salvage in the perimeter of the $\mathrm{MI}$ and the decrease in infarct size in the hUCBC in Gel Group can also be due, at least in part, to the increase in arteriole density. hUCBC secrete the growth factors vascular endothelial growth factor, fibroblastic growth factor, stem cell factor, placental growth factor, angiogenin, and angiopoietin that can contribute to myocardial neovascularization and accelerate infarct healing by decreasing inflammation, attracting native vascular endothelial progenitor cells or by stimulating the formation of new blood vessels from existing blood vessels in the perimeter of the damaged myocardium [50,51,60-62]. Neovascularization of ischemic muscle by stem cells can occur within two weeks [63-68].

\section{Conclusion}

The present investigation suggests that hUCBC in chitosan hydrogels can significantly decrease MI size, reduce adverse LV remodeling, and increase vascularization in the perimeter of a MI. As a consequence, LV contractility is increased. The anatomical and hemodynamic benefits of hUCBC in chitosan exceed the beneficial changes seen with hUCBC or chitosan alone. hUCBC plus chitosan gel studies are on-going in our cardiovascular research laboratory.

\section{Acknowledgment}

This work was sponsored, in part, by a grant from the Children's Cardiomyopathy Foundation and the Research Fund of the First Affiliated Hospital, Sun Yat-sen University, Guangzhou, China. These agencies had no role in the creation of the study design, data collection, analyses, interpretation of the data, or the writing of the manuscript. The authors thank the Florida Pathology Associates, and the Departments of Cardiology, Cardiothoracic Surgery, and Pathology, First Affiliated Hospital, Sun Yat-sen University for their generous assistance during this project.

\section{Disclosures}

Declarations of interest: None. The authors have no financial relationships with other people or organizations that could potentially and inappropriately influence their work and conclusions.

\section{References}

1. Benjamin E, Virani S, Callaway C, Chamberlain A, Chang A, et al. (2018) Heart disease and stroke statistics-2018 update: A report from the american heart association. Circulation 137: e67-e492.

2. Mummery C, Davis R, Krieger J (2010) Challenges in using stem cells for cardiac repair. Sci Transl Med 2.

3. Cohn J, Ferrari R, Sharpe N (2000) Cardiac remodeling-concepts and clinical implications: A consensus paper from an international forum on cardiac remodeling. Behalf of an international forum on cardiac remodeling. J Am Coll Cardiol 35: 569-582.

4. Henning RJ (2018) Current status of stem cells in cardiac repair. Future Cardiol 14: 181-192.

5. Traverse JH, Henry TD, Ellis SG, Pepine CJ, Willerson JT, et al. (2011) Effect of intracoronary delivery of autologous bone marrow mononuclear cells 2 to 3 weeks following acute myocardial infarction on left ventricular function: The LateTIME randomized trial. JAMA 306: 2110-2119.

6. Traverse JH, Henry TD, Pepine CJ, Willerson JT, Zhao DX, et al. (2012) Effect of use and timing of bone marrow mononuclear cell delivery on left ventricular function after acute myocardial infarction. The TIME randomized trial. JAMA 308: 2380-2389.

7. Sürder D, Manka R, Lo Cicero V, Moccetti T, Rufibach K, et al. (2013) Intracoronary injection of bone marrow derived mononuclear cells, early or late after acute myocardial infarction: Effects on global left ventricular function. Circulation 127: 1968-1979.

8. Henry T, Kereiakes D, Kowalchuk G, Aguirre F, Malliaris K, et al. (2017) ALLSTAR 6-month results of allogeneic heart stem cells to acheive myocardial regeneration (ALLSTAR) trial: A randomized placebo-controlled, double blind study.

9. Müller-Ehmsen J, Krausgrill B, Burst V, Schenk K, Neisen UC, et al. (2006) Effective engraftment but poor mid-term persistence of mononuclear and mesenchymal bone marrow cells in acute and chronic rat myocardial infarction. J Mol Cell Cardiol 41: 876-884.

10. van den Akker F, Feyen D, van den Hoogen p, van Laake L, van Eeuwijk EC, et al. (2017) Intramyocardial stem cell injection: Go(ne) with the flow. Eur Heart J 38: 184-186. 
11. Henning RJ, Abu-Ali H, Balis J, Morgan M, Willing A, et al. (2004) Human umbilical cord stem cells in the treatment of acute myocardial infarction. Cell Transplant 13: 729-739.

12. Henning RJ, Burgos J, Ondrovic L, Sanberg P, Balis J, et al. (2006) Human umbilical cord blood progenitor cells are attracted to infarcted myocardium and significantly reduce myocardial infarction size. Cell Transplant 15: 647-658.

13. Henning RJ, Burgos J, Vasko M, Alvarado F, Sanberg CD, et al. (2007) Human umbilical cord stem cells and myocardial infarction: Effect of dose and route of administration on infarct size. Cell Transplant 16: 907-917.

14. Henning RJ, Shariff M, Ujwala E, Alvarado F, Vasko M, et al. (2006) Human cord blood mononuclear cells decrease cytokines and inflammatory cells in acute infarcts. Stem Cells Dev 17: 1207-1220.

15. Henning RJ, Aufman J, Shariff M, Sawmiller D, DeLostia $V$, et al. (2010) Human umbilical cord stem cells decrease fibrosis and increase cardiac function in cardiomyopathy. Regen Med 5: 45-54.

16. Henning RJ, Khan A, Jimenez E (2016) Chitosan hydrogels significantly limit left ventricular infarction and remodeling and preserve myocardial contractility. J Surg Res 201: 490497.

17. Chenite A, Chaput C, Wang D, C Combesa, Buschmann MD, et al. (2000) Novel injectable solutions of chitosan form biodegradable gels in situ. Biomaterials 21: 2155-2161.

18. Dong W, Han B, Feng Y, Song F, Chang J, et al. (2010) Pharmacokinetics and biodegradation mechanisms of a versatile carboxymethyl derivative of chitosan: In vivo and in vitro evaluation. Biomacromolecules 11: 1527-1533.

19. Jang T, Kumbar S, Nair L, Laurencin C (2008) Biologically active chitosan systems for tissue engineering and regenerative medicine. Curr Top Med Chem 8: 354-364.

20. Rodrıquez-Vazquez M, Vega-Ruiz B, Ramos-Zuniga $R$, Saldan a-Koppel D, Quinones-Olvera L (2015) Chitosan and its potential use as a scaffold for tissue engineering in regenerative medicine. Biomed Res Int.

21. Ishihara M, Nakanishi K, Ono K, Sato M, Kikuchi M, et al. (2002) Photocrosslinkable chitosan as a dressing for wound occlusion and accelerator in healing process. Biomaterials 23: 833-840.

22. Hasan A, Khattab A, Islam MA, Hweij KA, Zeitouny J, et al. (2015) Injectable hydrogels for cardiac tissue repair after myocardial infarction. Adv Sci (Weinh) 2: 1500122

23. Sannino A, Demitri C, Madaghiele M (2009) Biodegradable cellulose based hydrogels: Design and applications. Materials (Basel) 2: 353-373.

24. Tomihata K, Ikada Y (1997) In vitro and in vivo degradation of films of chitin and its deacetylated derivatives. Biomaterials 18: $567-575$.

25. Adegboyega P, Adesokan A, Haque A, Boor P (1997) Sensitivity and specificity of triphenyl tetrazolium chloride in the gross diagnosis of acute myocardial infarcts. Arch Pahol Lab Med 121: 1063-1068.

26. Rossomacha E, Hoemann C, Shive M (2004) Simple methods for staining chitosan in biotechnological applications. Journal of Histotechnology 27: 31-36.

27. Azar A, Tavakoli F, Moladoust H, Zare A, Sadeghpour A (2014) Echocardiographic evaluation of cardiac function in ischemic rats: Value of m-mode echocardiography. Res Cardiovasc Med 3: e22941.
28. Morgan E, Faullx M, McElfresh T, Kung T, Zawaneh MS, et al. (2004) Validation of echocardiographic methods for assessing left ventricular dysfunction in rats with myocardial infarction. Am J Physiol Heart Circ Physiol 287: 2049-2053.

29. Muzzarelli R (1997) Human enzymatic activities related to the therapeutic administration of chitin derivatives. Cell Mol Life Sci 53: 131-140.

30. Kurdi M, Chidiac R, Hoemann C, Zouein F, Zgheib C, et al. (2010) Hydrogels as platforms for stem cells delivery to the heart. Congest Heart Fail 16: 132-135.

31. Ruvinov E, Sapir Y, Cohen S (2012) Cardiac tissue engineering: Principles, materials and applications. ( $1^{\text {st }}$ edn), Synthesis lectures on tissue engineering, 1-200.

32. Lu WN, Lü SH, Wang HB, Li DX, Duan CM, et al. (2009) Functional improvement of infarcted heart by co-injection of embryonic stem cells with temperature-responsive chitosan hydrogel. Tissue Eng Part A 15: 1437-1447.

33. Wall S, Walker J, Healy K, Ratcliffe M, Guccione J (2006) Theoretical impact of the injection of material into the myocardium: A finite element model simulation. Circulation 114: $2621-2635$.

34. Kichula E, Wang H, Dorsey S, Szczesny SE, Elliott DM, et al. (2014) Experimental and computational investigation of altered mechanical properties in myocardium after hydrogel injection. Ann Biomed Eng 42: 1546-1556.

35. Deng C, Zhang P, Vulesevic B, Kuraitis D, Li F, et al. (2010) A collagen-chitosan hydrogel for endothelial differentiation and angiogenesis. Tissue Eng Part A 16: 3099-3109.

36. Chupa J, Foster A, Sumner S, Madihally S, Matthew H (2000) Vascular cell responses to polysaccharide materials: In vitro and in vivo evaluations. Biomaterials 21: 2315-2322.

37. Ishihara M, Ono K, Sato M, Nakanishi K, Saito $Y$, et al. (2001) Acceleration of wound contraction and healing with a photocrosslinkable chitosan hydrogel. Wound Repair Regen 9: 513-521.

38. Mori T, Okumura M, Matsuura M, Ueno K, Tokura S, et al. (1997) Effects of chitin and its derivatives on the proliferation and cytokine production of fibroblasts in vitro. Biomaterials 18: 947-951.

39. Fischer TH, Thatte HS, Nichols TC, Bender-Neal DE, Bellinger AD, et al. (2005) Synergistic platelet integrin signaling and factor XII activation in poly-N-acetyl glucosamine fiber-mediated hemostasis. Biomaterials 26: 5433-5443

40. Liu Z, Wang H, Wang Y, Lin Q, Yao A, et al. (2012) The influence of chitosan hydrogel on stem cell engraftment, survival and homing in the ischemic myocardial microenvironment. Biomaterials 33: 3093-3106.

41. Madihally S, Matthew H (1999) Porous chitosan scaffolds for tissue engineering. Biomaterials 20: 1133-1142.

42. Xu B, Li Y, Deng B, Liu X, Wang L, et al. (2017) Chitosan hydrogel improves mesenchymal stem cell transplant survival and cardiac function following myocardial infarction in rats. Exp Ther Med 13: 588-594.

43. Ueno $\mathrm{H}$, Murakami M, Okumura M, Kadosawa $\mathrm{T}$, Uede $\mathrm{T}$, et al. (2001) Chitosan accelerates the production of osteopontin from polymorphonuclear leukocytes. Biomaterials 22: $1667-$ 1673.

44. Dai W, Hale S, Kay G, Jyrala A, Kloner R (2009) Delivering stem cells to the heart in a collagen matrix reduces relocation of cells to other organs as assessed by nanoparticle Technology. Regen Med 4: 387-395. 
45. Hal E Broxmeyer (2010) Cord blood hematopoietic stem cell transplantation. In: Hal E Broxmeyer, Stem book. Harvard Stem Cell Institute, Cambridge (MA), USA.

46. Broxmeyer HE, Lee MR, Hangoc G, Cooper S, Prasain N, et al. (2011) Hematopoietic stem/progenitor cells, generation of induced pluripotent stem cells, and isolation of endothelial progenitors from 21- to 23.5-year cryopreserved cord blood. Blood 117: 4773-4777.

47. Wagner J, Rosenthal J, Sweetman R, Shu X, Davies SM, et al. (1996) Successful transplantation of HLA-matched and HLA mismatched umbilical cord blood from unrelated donors. Analysis of engraftment and acute graft versus host disease. Blood 88: 795-802.

48. Zhang J, Chen G, Wang $\mathrm{Y}$, Zhao J, Duan $\mathrm{H}$, et al. (2012) Hydrogen peroxide preconditioning enhances the therapeutic efficacy of Wharton's Jelly mesenchymal stem cells after myocardial infarction. Chin Med J (Engl) 125: 3472-3478.

49. Sasaki K, Bannai S, Makino N (1998) Kinetics of hydrogen peroxide elimination by human umbilical vein endothelial cells in culture. Biochim Biophys Acta 1380: 275-288.

50. Jin H, Sanberg P, Henning RJ (2013) Human umbilical cord blood mononuclear cell conditioned media inhibits hypoxicinduced apoptosis in human coronary artery endothelial cells and cardiac myocytes by activation of the survival proteins Akt. Cell Transplant 22: 1637-1650.

51. Henning RJ, Sanberg P, Jimenez E (2014) Human cord blood stem cell paracrine factors activate the survival protein kinase Akt and inhibit death protein kinases JNK and p38 in injured cardiomyocytes. Cytotherapy 16: 1158-1168.

52. Zhao Y, Huang Z, Qi M, Lazzarini P, Mazzone T (2007) Immune regulation of $T$ lymphocyte by a newly characterized human umbilical cord blood stem cell. Immunol Lett 108: 78-87.

53. Henning RJ (2016) Therapeutic angiogenesis: Angiogennic growth factors for ischemic heart disease. Future Cardiol 12: $585-599$.

54. Guo H, Wang H, Tan Y, Wu J (2011) Transplantation of marrow-derived cardiac stem cells carried in fibrin improves cardiac function after myocardial infarction. Tissue Eng Part A 17: 45-58.

55. Bhutani S, Nachlas A, Brown M, Pete T, Johnson CT, et al. (2018) Evaluation of hydrogels presenting extracellular matrix-derived adhesion peptides and encapsulating cardiac progenitor cells for cardiac repair. ACS Biomater Sci Eng 4: 200-210.

56. Fujita M, Ishihara M, Morimoto $Y$, Simizu M, Saito $Y$, et al. (2005) Efficacy of photocrosslinkable chitosan hydrogel containing fibroblast growth factor-2 in a rabbit model of chronic myocardial infarction. J Surg Res 126: 27-33.
57. Hashizume R, Fujimoto KL, Hong Y, Guan J, Toma C, et al. (2013) Biodegradable elastic patch plasty ameliorates left ventricular adverse remodeling after ischemia-reperfusion injury: A preclinical study of a porous polyurethane material in a porcine model. J Thorac Cardiovasc Surg 146: 391399.

58. Howling G, Dettmar P, Goddard P, Hamson F, Dornish $M$, et al. (2001) The effect of chitin and chitosan on the proliferation of human skin fibroblasts and keratinocytes in vitro. Biomaterials 22: 2959-2966.

59. Patrulea V, Ostafe V, Borchard G, Jordan O (2015) Chitosan as a starting material for wound healing applications. Eur $\mathrm{J}$ Pharm Biopharm 97: 417-426.

60. Moon K, Lee J, Han S, Lee H, Dhong E (2017) Effects of human umbilical cord blood-derived mesenchymal stromal cells and dermal fibroblasts on diabetic wound healing. Cytotherapy 19: 821-828.

61. Hu Y, Rao SS, Wang ZX, Cao J, Tan YJ, et al. (2018) Exosomes from human umbilical cord blood accelerate cutaneous wound healing through miR-21-3p-mediated promotion of angiogenesis and fibroblast function. Theranostics 8: 169-184.

62. Zhang J, Chen C, Hu B, Niu X, Liu X, et al. (2016) Exosomes derived from human endothelial progenitor cells accelerate cutaneous wound healing by promoting angiogenesis through Erk1/2 signaling. Int J Biol Sci 12: 1472-1487.

63. Asahara, T, Masuda H, Tomono T, Kalka C, Pastore C, et al. (1999) Bone marrow origin of endothelial progenitor cells responsible for postnatal vasculogenesis in physiological and pathological neovascularization. Circ Res 85: 221-228.

64. Gojo S, Gojo N, Takeda Y, Mori T, Abe H, et al. (2003) In vivo cardiovasculogenesis by direct injection of isolated adult mesenchymal stem cells. Exp Cell Res 288: 51-59.

65. Kinnaird T, Stabile E, Burnett M, Shou M, Lee C, et al. (2004) Local delivery of marrow-derived stromal cells augments collateral perfusion through paracrine mechanisms. Circulation 109: 1543-1549.

66. Moon M, Kim S, Kim Y, Kim S, Lee J, et al. (2006) Human adipose tissue-derived mesenchymal stem cells improve postnatal neovascularization in a mouse model of hindlimb ischemia. Cell Physiol Biochem 17: 279-290.

67. Usui S, Iso Y, Sasai M, Mizukami T, Sato C, et al. (2014) Mesenchymal stem cells from bone marrow enhance neovascularization and stromal cell proliferation in rat ischemic limb in the earl phase after implantation. Showa Univ J Med Sci 26: 121-129.

68. Vicario J, Piva J, Pierini A, Ortega HH, Canal A, et al. (2002) Transcoronary sinus delivery of autologous bone marrow and angiogenesis in pig models with myocardial injury. Cardiovasc Radiat Med 3: 91-94. 